\title{
Prognostic Implications of the Predominant
} Histologic Subtype in Stage I Lung Adenocarcinoma: Lack of Association with Overall
Survival Based on Multi-Aspect Survival Analyses

Jong Hyuk Lee

Seoul National University Hospital

Hyungjin Kim ( $\boldsymbol{\nabla}$ khj.snuh@gmail.com )

Seoul National University Hospital

Chang Min Park

Seoul National University Hospital

Yoon Kyung Jeon

Seoul National University Hospital

Doo Hyun Chung

Seoul National University Hospital

Young Tae Kim

Seoul National University College of Medicine

Jin Mo Goo

Seoul National University

\section{Research Article}

Keywords: adenocarcinoma, histologic subtype, overall survival, survival analysis, cure model

Posted Date: February 18th, 2022

DOI: https://doi.org/10.21203/rs.3.rs-1322231/v1

License: (a) (i) This work is licensed under a Creative Commons Attribution 4.0 International License.

Read Full License 


\section{Abstract}

We aimed to investigate the prognostic role of the predominant histologic subtype classification of lung adenocarcinoma in the era of the eighth-edition staging system. A total of 587 patients with stage I adenocarcinomas (lepidic, $n=46$; acinar/papillary, $n=484$; solid/micropapillary, $n=57$ ) were assessed. The prognostic value of this subtype classification for overall survival (OS) was investigated with three multivariable analyses adjusted for the eighth-edition clinical T category and radiologic nodule type: the adjusted log-rank test, multivariable Cox hazard regression, and multivariable mixture cure model to evaluate long-term and short-term prognostic associations separately. In the adjusted log-rank test for pairwise comparisons, no evidence was found for differences in OS among the subtype classifications $(p>.05)$. The histologic subtype was not an independent prognostic factor in either the Cox regression analysis (hazard ratio [HR] of acinar/papillary subtypes: 3.03; 95\% confidence intertval [CI]: 0.4-22.59; $\mathrm{p}=0.28$; HR of solid/micropapillary subtypes: $2.78 ; 95 \% \mathrm{Cl}: 0.34-22.37 ; \mathrm{p}=.34$ ) or the mixture cure model (cure probability model; odds ratio: 1.06 [95\% Cl: 0.38-2.95; $\mathrm{p}=.91$ ]; failure time distribution model; HR: 0.94 [95\% Cl: $0.36-2.44 ; p=.89])$. The prognostic implication of the predominant histologic subtype classification is not clear for stage I lung adenocarcinoma in the eighth-edition staging system.

\section{Introduction}

Lung cancer is the leading cause of cancer mortality and second most common newly diagnosed type of cancer worldwide and in the United States. ${ }^{1,2}$ The most common histologic type of lung cancer is adenocarcinoma, accounting for up to $60 \%$ of cases, and this single histologic type has a wide spectrum of clinical, radiologic, and pathologic characteristics. ${ }^{3,4}$ According to the 2011 International Association for the Study of Lung Cancer/American Thoracic Society/European Respiratory Society (IASLC/ATS/ERS) classification, invasive lung adenocarcinoma is histologically categorized into lepidic, acinar, papillary, solid, and micropapillary subtypes based on the predominant growth pattern. ${ }^{3}$ Many studies have reported the prognostic value of this classification system. ${ }^{4-22}$ Specifically, adenocarcinoma of the lepidic-predominant subtype showed the most favorable outcomes, followed by the acinar/papillary and solid/micropapillary subtypes. $4,5,7,9-11,13-17,19,20,22,23$ The value of this prognostic stratification was proven in early-stage adenocarcinoma and in advanced disease. ${ }^{4-11,13-20,22,23}$

The staging system by the American Joint Commission on Cancer (AJCC) is the cornerstone for predicting the prognosis of patients with lung cancer. ${ }^{24}$ The eighth-edition staging system adopted the solid portion size on CT and the invasive component size on microscopy to determine the clinical and pathologic T category, respectively. ${ }^{25,26}$ It is now well established that the solid portion size or invasive component size is a better prognostic predictor than the total tumor size. ${ }^{25,26}$ In this context, the prognostic value of the predominant adenocarcinoma subtype has not yet been investigated in conjunction with the eighth-edition staging system. Therefore, the independent prognostic implication of histologic subtypes for early-stage adenocarcinoma in the era of the eighth-edition staging system remains unclear. 
In this study, we aimed to validate the prognostic value of the predominant histologic subtype in patients with resected stage I adenocarcinoma using multi-aspect survival analyses, including the adjusted logrank test, Cox proportional hazard regression, and mixture cure model to distinguish short-term and longterm prognoses.

\section{Results}

\section{Demographic and Radiologic Characteristics}

The clinical and radiologic characteristics of the study population and subgroups according to the histologic subtypes are described in Table 1. Of the 587 patients (334 women; median age, $64 \mathrm{y}$ ), 46 (7.8\%), 484 (82.5\%), and 57 patients (9.7\%) had adenocarcinomas of the lepidic, acinar/papillary, and solid/micropapillary-predominant subtypes, respectively. Among these three groups, significant differences in sex $(p=0.009)$, smoking status $(p=0.001)$, nodule type $(p<0.001)$, and clinical T category $(p<0.001)$ were noted. In particular, part-solid nodules and an earlier clinical T category (cT1mi and cT1a) were more frequently observed in the lepidic subtype (part-solid nodule: $87 \%$; earlier clinical T category: $60.9 \%$ ) than in the acinar/papillary (part-solid nodule: $48.6 \%$; earlier clinical T category: $14.5 \%$ ) and solid/micropapillary-predominant subtypes (part-solid nodule: 7\%; earlier clinical T category: 0\%) (Fig. 1). The median interval between the preoperative CT scan and surgery was 21 days (interquartile range [IQR]: 3-32 days), and the median follow-up duration after surgery was 81.3 months (IQR: 68.7-100.3 months). 
Table 1

Clinical and Radiologic Characteristics of the Study Population

Predominant pathological subtype of adenocarcinoma

\begin{tabular}{|c|c|c|c|c|c|}
\hline Variable & $\begin{array}{l}\text { Total } \\
(n=587)\end{array}$ & $\begin{array}{l}\text { Lepidic } \\
(n=46)\end{array}$ & $\begin{array}{l}\text { Acinar/Papillary } \\
(n=484)\end{array}$ & $\begin{array}{l}\text { Solid/Micropapillary } \\
(n=57)\end{array}$ & $\mathrm{p}$ Value \\
\hline Age (years)* & $\begin{array}{l}64(56- \\
71)\end{array}$ & $\begin{array}{l}61(53- \\
69)\end{array}$ & $64(56-71)$ & $66(60-71)$ & 0.13 \\
\hline Sex & & & & & 0.009 \\
\hline Male & $\begin{array}{l}253 \\
(43.1)\end{array}$ & $22(47.8)$ & $196(40.5)$ & 35 (61.4) & \\
\hline Female & $\begin{array}{l}334 \\
(56.9)\end{array}$ & $24(52.2)$ & $288(59.5)$ & $22(38.6)$ & \\
\hline $\begin{array}{l}\text { History of } \\
\text { malignancy other } \\
\text { than lung cancer }\end{array}$ & & & & & 0.24 \\
\hline No & $\begin{array}{l}491 \\
(83.6)\end{array}$ & $35(76.1)$ & $410(84.7)$ & $46(80.7)$ & \\
\hline Yes & $96(16.4)$ & $11(23.9)$ & $74(15.3)$ & $11(19.3)$ & \\
\hline Smoking status & & & & & 0.001 \\
\hline Never smoker & $\begin{array}{l}372 \\
(63.4)\end{array}$ & $32(69.6)$ & $317(65.5)$ & $23(40.4)$ & \\
\hline Current or ex-smoker & $\begin{array}{l}215 \\
(36.6)\end{array}$ & $14(30.4)$ & $167(34.5)$ & $34(59.6)$ & \\
\hline Nodule type & & & & & $<0.001$ \\
\hline Part-solid nodule & $\begin{array}{l}279 \\
(47.5)\end{array}$ & $40(87)$ & $235(48.6)$ & $4(7)$ & \\
\hline Solid nodule & $\begin{array}{l}308 \\
(52.5)\end{array}$ & $6(13)$ & $249(51.4)$ & $53(93)$ & \\
\hline Lobar location & & & & & 0.13 \\
\hline
\end{tabular}

Data are numbers of patients with percentages in parentheses, unless otherwise specified.

*Data are median values with the interquartile range in parentheses.

†The clinical T categorization followed the eighth-edition staging system for lung cancer.

¥The pathologic $T$ categorization according to the seventh-edition staging system.

$\S T$ The comparison was performed with the categories collapsed into four (cT1 mi/cT1a, cT1b, cT1c, and cT2). 


\section{Predominant pathological subtype of adenocarcinoma}

\begin{tabular}{lllll} 
Upper lobe & $\begin{array}{l}350 \\
(57.9)\end{array}$ & $31(67.4)$ & $271(56)$ & $38(66.7)$ \\
\hline Other lobe & $\begin{array}{l}247 \\
(42.1)\end{array}$ & $15(32.6)$ & $213(44)$ & $19(33.3)$ \\
\hline
\end{tabular}

Clinical T category $\dagger$

$<0.001 \S$

\begin{tabular}{|c|c|c|c|c|}
\hline cT1mi & $20(3.4)$ & 7 (15.2) & $13(2.7)$ & $0(0)$ \\
\hline cT1a & 78 (13.3) & $21(45.7)$ & $57(11.8)$ & $0(0)$ \\
\hline cT1b & $\begin{array}{l}261 \\
(44.4)\end{array}$ & 15 (32.6) & $221(45.7)$ & $25(43.9)$ \\
\hline cT1c & $\begin{array}{l}169 \\
(28.8)\end{array}$ & $3(6.5)$ & $144(29.8)$ & $22(38.6)$ \\
\hline cT2 & 59 (10.1) & $0(0)$ & 49 (10.1) & $10(17.5)$ \\
\hline
\end{tabular}

Pathological T categorył

pT1a

206

(35.1)

$26(56.5) \quad 159(32.9)$

$21(36.8)$

pT1b

124

(21.1)

$12(26.1) \quad 99(20.5)$

$13(22.8)$

pT2a

257

(43.8)

$8(17.4) \quad 226(46.7)$

$23(40.4)$

CT-to-surgery

interval (days)*

21 (3-

32)

22.5

(2.0-

$21.0(4.5-32.0)$

$19.0(2.0-31.0)$

0.60

34.0)

Follow-up interval

(months)*

81.3

(68.7-

100.3)

80.1

(69.0-

100.1)

$80.1(69.0-$

100.1)

Survival status

Alive

502

(85.5)

$45(97.8) \quad 414(85.5)$

$43(75.4)$

Data are numbers of patients with percentages in parentheses, unless otherwise specified.

*Data are median values with the interquartile range in parentheses.

†The clinical T categorization followed the eighth-edition staging system for lung cancer.

¥The pathologic $T$ categorization according to the seventh-edition staging system.

$\S T$ The comparison was performed with the categories collapsed into four (cT1 mi/cT1a, cT1b, cT1c, and cT2). 


\begin{tabular}{|c|c|c|}
\hline \multicolumn{3}{|c|}{ Predominant pathological subtype of adenocarcinoma } \\
\hline $85(14.5)$ & $70(14.5)$ & $14(24.6)$ \\
\hline \multicolumn{3}{|c|}{ Data are numbers of patients with percentages in parentheses, unless otherwise specified. } \\
\hline \multicolumn{3}{|c|}{ *Data are median values with the interquartile range in parentheses. } \\
\hline \multicolumn{3}{|c|}{ †The clinical T categorization followed the eighth-edition staging system for lung cancer. } \\
\hline \multicolumn{3}{|c|}{$\ddagger$ †Te pathologic T categorization according to the seventh-edition staging system. } \\
\hline $\begin{array}{l}\text { §The comparison was performed } \\
\text { and cT2). }\end{array}$ & the categories collap & ur (cT1mi/cT1a, cT1b, cT1c, \\
\hline
\end{tabular}

\section{Overall Survival According to the Histologic Subtype}

The 5-year OS rates were $97.8 \%$ (45 of 46 ; $95 \%$ Cl: $93.7-100 \%$ ) for the lepidic subtype, $91.3 \%$ (414 of 484 ; $95 \% \mathrm{Cl}: 88.9-93.9 \%$ ) for the acinar/papillary subtype, and $86 \%$ (43 of $57 ; 95 \% \mathrm{Cl}: 77.4-95.5 \%$ ) for the solid/micropapillary-predominant subtype (Table 2; Fig. 2). For the solid tumor subgroup, the 5-year OS rates were $100 \%$ ( 6 of $6 ; 95 \% \mathrm{Cl}: 100-100 \%$ ) for the lepidic subtype, $88 \%$ (203 of $249 ; 95 \%$ Cl: $84-92.1 \%$ ) for the acinar/papillary subtype, and $84.9 \%$ (41 of $53 ; 95 \% \mathrm{Cl}: 75.8-95.1 \%$ ) for the solid/micropapillarypredominant subtype (Table 2). 
Table 2

Unadjusted and Adjusted Pairwise Comparisons Using the Log-rank Test for Overall Survival

\begin{tabular}{|c|c|c|c|c|c|c|}
\hline $\begin{array}{l}\text { Study } \\
\text { population }\end{array}$ & $\begin{array}{l}\text { Predominant } \\
\text { histologic subtype of } \\
\text { adenocarcinoma }\end{array}$ & $\begin{array}{l}\text { Number of } \\
\text { events per } \\
\text { total patients }\end{array}$ & $\begin{array}{l}\text { Median } \\
\text { OS rate } \\
(\%)\end{array}$ & $\begin{array}{l}5 \text {-year } \\
\text { OS } \\
\text { rate } \\
(\%)\end{array}$ & $\begin{array}{l}p \text { Value } \\
\text { (unadjusted } \\
\text { log-rank } \\
\text { test) }\end{array}$ & $\begin{array}{l}p \text { Value } \\
\text { (adjusted } \\
\text { log-rank } \\
\text { test) }\end{array}$ \\
\hline \multirow[t]{3}{*}{ Total } & Lepidic & $1 / 46$ & $\mathrm{~N} / \mathrm{A}$ & $\begin{array}{l}97.8 \\
(93.7 \\
100)\end{array}$ & & \\
\hline & Acinar/papillary & $70 / 484$ & $\mathrm{~N} / \mathrm{A}$ & $\begin{array}{l}91.3 \\
(88.9 \\
93.9)\end{array}$ & $0.045^{\star}$ & $0.44^{\star}$ \\
\hline & Solid/micropapillary & $14 / 57$ & $\mathrm{~N} / \mathrm{A}$ & $\begin{array}{l}86.0 \\
(77.4 \\
95.5)\end{array}$ & $0.02 \dagger$ & $0.40 \dagger$ \\
\hline \multirow[t]{3}{*}{$\begin{array}{l}\text { Solid } \\
\text { tumor } \\
\text { subgroup }\end{array}$} & Lepidic & $0 / 6$ & $\mathrm{~N} / \mathrm{A}$ & $\begin{array}{l}100 \\
(100 \\
100)\end{array}$ & & \\
\hline & Acinar/papillary & $46 / 249$ & $\mathrm{~N} / \mathrm{A}$ & $\begin{array}{l}88.0 \\
(84.0 \\
92.1)\end{array}$ & & \\
\hline & Solid/micropapillary & $12 / 53$ & $\mathrm{~N} / \mathrm{A}$ & $\begin{array}{l}84.9 \\
(75.8 \\
95.1)\end{array}$ & $0.70 \neq$ & $0.74 \ddagger$ \\
\hline \multicolumn{7}{|c|}{${ }^{\star} p$ value for the comparison between the lepidic and acinar/papillary-predominant subtypes. } \\
\hline \multicolumn{7}{|c|}{$\begin{array}{l}+p \text { value for the comparison between the lepidic and solid/micropapillary-predominant subtypes. The } \\
\text { comparison between the acinar/papillary and solid/micropapillary subtypes demonstrated } p \text { values } \\
\text { of } 0.13 \text { for the unadjusted log-rank test and } 0.72 \text { for the adjusted log-rank test. }\end{array}$} \\
\hline \multicolumn{7}{|c|}{$\begin{array}{l}\ddagger p \text { value for the comparison between the lepidic/acinar/papillary-predominant and } \\
\text { solid/micropapillary-predominant subtypes. }\end{array}$} \\
\hline
\end{tabular}

The unadjusted log-rank test exhibited longer survival for the lepidic subtype than for the acinar/papillary subtype ( $p=0.045)$ and solid/micropapillary subtype ( $p=0.02)$ (Table 2; Fig. 2.). However, after adjustment for prognostic factors, including the clinical $T$ category and nodule type, the comparisons did not reach statistical significance ( $p=0.44$ and $p=0.40$, respectively; Table 2; Fig. 2). For solid nodules, there were no significant differences in OS between the lepidic/acinar/papillary and solid/micropapillary-predominant subtypes in both the unadjusted log-rank $(p=0.70)$ and adjusted log-rank tests $(p=0.74)$ (Table 2; Supplementary Fig. 1).

\section{Multivariable Cox Proportional Hazard Regression Analysis}


The multivariable Cox proportional hazard regression model revealed that the histologic subtype of adenocarcinoma was not an independent predictor for OS (HR of the acinar/papillary subtype: 3.03; 95\% Cl: $0.4-22.59 ; p=0.28$; HR of the solid/micropapillary subtype: $2.78 ; 95 \% \mathrm{Cl}: 0.34-22.37 ; p=0.34 ;$ Table 3 ). When the lepidic/acinar/papillary-predominant subtypes were combined into the reference category because of the small number of cases and rarity of deaths in patients with the lepidic-predominant subtype, the solid/micropapillary subtype was also not an independent predictor (HR: 0.92; 95\% Cl: 0.511.69; $p=0.80$; Supplementary Table 1). Regarding only solid nodules, the histologic subtype was likewise not associated with OS (HR of the solid/micropapillary subtype: $0.86 ; 95 \% \mathrm{Cl}: 0.45-1.65 ; p=0.65$; Supplementary Table 2). 
Table 3

Multivariable Cox Proportional Hazard Regression Analysis for Overall Survival

\begin{tabular}{|c|c|c|c|}
\hline Variable & Subcategory & $\begin{array}{l}\mathrm{HR} \\
(95 \% \\
\mathrm{Cl})\end{array}$ & Value \\
\hline Age (year) & & $\begin{array}{l}1.05 \\
(1.03 \\
1.08)\end{array}$ & $<0.001$ \\
\hline Women (reference: men) & & $\begin{array}{l}0.72 \\
(0.35 \\
1.49)\end{array}$ & 0.37 \\
\hline $\begin{array}{l}\text { History of malignancy other than lung cancer (reference: } \\
\text { no history of malignancy) }\end{array}$ & & $\begin{array}{l}1.85 \\
(1.1 \\
3.11)\end{array}$ & 0.02 \\
\hline Ex- or current smoker (reference: never-smoker) & & $\begin{array}{l}1.62 \\
(0.8, \\
3.28)\end{array}$ & 0.18 \\
\hline Solid nodule (reference: part-solid nodule) & & $\begin{array}{l}1.26 \\
(0.76 \\
2.09)\end{array}$ & 0.37 \\
\hline \multirow[t]{3}{*}{$\begin{array}{l}\text { Clinical T category } \\
\text { (reference: cT1mi/cT1a) }\end{array}$} & cT1b & $\begin{array}{l}2.64 \\
(0.78 \\
8.92)\end{array}$ & 0.12 \\
\hline & cT1c & $\begin{array}{l}3.65 \\
(1.04 \\
12.86)\end{array}$ & 0.044 \\
\hline & cT2 & $\begin{array}{l}6.92 \\
(1.89 \\
25.32)\end{array}$ & 0.003 \\
\hline \multirow[t]{2}{*}{$\begin{array}{l}\text { Adenocarcinoma subtype } \\
\text { (reference: lepidic) }\end{array}$} & Acinar/papillary & $\begin{array}{l}3.03 \\
(0.4 \\
22.59)\end{array}$ & 0.28 \\
\hline & Solid/micropapillary & $\begin{array}{l}2.78 \\
(0.34 \\
22.37)\end{array}$ & 0.34 \\
\hline
\end{tabular}

Mixture Cure Model for Overall Survival

In the cure probability model, the predominant histologic subtype was not associated with long-term survival (OR of the solid/micropapillary subtype: $1.06 ; 95 \% \mathrm{Cl}: 0.38-2.95 ; p=0.91$ ). In the failure time distribution model, the histologic subtype was also not a prognostic factor for OS (HR of the solid/micropapillary subtype: $0.94 ; 95 \% \mathrm{Cl}: 0.36-2.44 ; p=0.89$ ) (Table 4 ). 
Table 4

Multivariable Mixture Cure Models for Overall Survival

\begin{tabular}{|c|c|c|c|c|}
\hline Model & Variable & Subcategory & $\begin{array}{l}\text { OR } \\
(95 \% \\
\text { Cl) }\end{array}$ & Value \\
\hline \multirow[t]{8}{*}{$\begin{array}{l}\text { tCure } \\
\text { probability } \\
\text { model }\end{array}$} & Age (year) & & $\begin{array}{l}0.93 \\
(0.90 \\
0.98)\end{array}$ & 0.002 \\
\hline & Women (reference: men) & & $\begin{array}{l}1.11 \\
(0.28 \\
4.36)\end{array}$ & 0.88 \\
\hline & $\begin{array}{l}\text { History of malignancy other than lung } \\
\text { cancer (reference: no history of } \\
\text { malignancy) }\end{array}$ & & $\begin{array}{l}0.36 \\
(0.14 \\
0.91)\end{array}$ & 0.03 \\
\hline & $\begin{array}{l}\text { Ex- or current smoker (reference: never- } \\
\text { smoker) }\end{array}$ & & $\begin{array}{l}0.33 \\
(0.08 \\
1.34)\end{array}$ & 0.12 \\
\hline & $\begin{array}{l}\text { Solid nodule (reference: part-solid } \\
\text { nodule) }\end{array}$ & & $\begin{array}{l}0.87 \\
(0.36 \\
2.12)\end{array}$ & 0.77 \\
\hline & $\begin{array}{l}\text { Clinical T category (reference: } \\
\text { cT1mi/cT1a/cT1b) }\end{array}$ & cT1c & $\begin{array}{l}0.37 \\
(0.16 \\
0.88)\end{array}$ & 0.02 \\
\hline & & cT2 & $\begin{array}{l}0.27 \\
(0.08 \\
0.90)\end{array}$ & 0.03 \\
\hline & $\begin{array}{l}\text { Adenocarcinoma subtype (reference: } \\
\text { lepidic/acinar/papillary) }\end{array}$ & Solid/micropapillary & $\begin{array}{l}1.06 \\
(0.38 \\
2.95)\end{array}$ & 0.91 \\
\hline Model & Variable & Subcategory & $\begin{array}{l}\text { HR } \\
(95 \% \\
\text { Cl) }\end{array}$ & $\mathrm{p}$ Value \\
\hline $\begin{array}{l}\text { ‡Failure time } \\
\text { distribution } \\
\text { model }\end{array}$ & Age (year) & & $\begin{array}{l}1.00 \\
(0.96 \\
1.05)\end{array}$ & 0.89 \\
\hline
\end{tabular}

$\mathrm{Cl}=$ confidence interval; $\mathrm{HR}=$ hazard ratio; $\mathrm{OR}=$ odds ratio

†The cure probability model is one of two components of the mixture cure model. For patients who have long-term survival, this model allows investigators to evaluate which covariates are associated with their long-term survival.

$\ddagger$ The failure time distribution model is another component of the mixture cure model. For patients who do not show long-term survival, this model allows investigators to evaluate which covariates are survival predictors. 


\begin{tabular}{|c|c|c|c|c|}
\hline Model & Variable & Subcategory & $\begin{array}{l}\text { OR } \\
(95 \% \\
\text { Cl) }\end{array}$ & Value \\
\hline & \multicolumn{2}{|l|}{ Women (reference: men) } & $\begin{array}{l}0.49 \\
(0.07 \\
3.48)\end{array}$ & 0.47 \\
\hline & \multicolumn{2}{|l|}{$\begin{array}{l}\text { History of malignancy other than lung } \\
\text { cancer (reference: no history of } \\
\text { malignancy) }\end{array}$} & $\begin{array}{l}0.77 \\
(0.26 \\
2.31)\end{array}$ & 0.64 \\
\hline & \multicolumn{2}{|l|}{$\begin{array}{l}\text { Ex-or current smoker (reference: never- } \\
\text { smoker) }\end{array}$} & $\begin{array}{l}0.45 \\
(0.07 \\
2.86)\end{array}$ & 0.40 \\
\hline & \multicolumn{2}{|l|}{$\begin{array}{l}\text { Solid nodule (reference: part-solid } \\
\text { nodule) }\end{array}$} & $\begin{array}{l}1.37 \\
(0.43 \\
4.39)\end{array}$ & 0.60 \\
\hline & \multirow[t]{2}{*}{$\begin{array}{l}\text { Clinical T category (reference: } \\
\text { cT1mi/cT1a/cT1b) }\end{array}$} & cT1c & $\begin{array}{l}0.67 \\
(0.24 \\
1.83)\end{array}$ & 0.43 \\
\hline & & cT2 & $\begin{array}{l}1.55 \\
(0.37 \\
6.42)\end{array}$ & 0.55 \\
\hline & $\begin{array}{l}\text { Adenocarcinoma subtype (reference: } \\
\text { lepidic/acinar/papillary) }\end{array}$ & Solid/micropapillary & $\begin{array}{l}0.94 \\
(0.36 \\
2.44)\end{array}$ & 0.89 \\
\hline \multicolumn{5}{|c|}{$\mathrm{Cl}=$ confidence interval; $\mathrm{HR}=$ hazard ratio; $\mathrm{OR}=$ odds ratio } \\
\hline \multicolumn{5}{|c|}{$\begin{array}{l}\text { The cure probability model is one of two components of the mixture cure model. For patients who } \\
\text { have long-term survival, this model allows investigators to evaluate which covariates are associated } \\
\text { with their long-term survival. }\end{array}$} \\
\hline \multicolumn{5}{|c|}{$\begin{array}{l}\text { ‡The failure time distribution model is another component of the mixture cure model. For patients } \\
\text { who do not show long-term survival, this model allows investigators to evaluate which covariates are } \\
\text { survival predictors. }\end{array}$} \\
\hline
\end{tabular}

Regarding solid nodules, the histologic subtype was not a predictor in either the cure probability model (OR of the solid/micropapillary subtype: $1.29 ; 95 \% \mathrm{Cl}: 0.51-3.25 ; p=0.60$ ) or the failure time distribution model (HR of solid/micropapillary subtype: $1.06 ; 95 \%$ Cl: 0.32-3.5; $p=0.92)$ (Supplementary Table 3 ).

\section{Sensitivity analysis}

After excluding those with mixed subtype adenocarcinoma $(n=153), 434$ adenocarcinomas were included in the sensitivity analysis. The 5-year OS rates were $97.8 \%$ (45 of $46 ; 95 \% \mathrm{Cl}$ : $93.7-100 \%$ ) for the lepidic subtype, $90 \%$ (306 of $359 ; 95 \% \mathrm{Cl}: 86.9-93.1 \%$ ) for the acinar/papillary subtype, and $82.8 \%$ (22 of 29; 95\% Cl: 70.1-97.7\%) for the solid/micropapillary-predominant subtype (Supplementary Table 4). 
The adjusted log-rank test did not show longer survival for the lepidic subtype than for the acinar/papillary subtype $(p=0.45)$ and solid/micropapillary subtype $(p=0.63)$ (Supplementary Table 4; Supplementary Fig. 2). The histologic subtype was not an independent predictor in either the Cox regression analysis (HR of solid/micropapillary subtypes: $0.98 ; 95 \% \mathrm{Cl}$ : 0.43-2.22; $p=0.96$; Supplementary Table 5) or the mixture cure model (cure probability model; OR of solid/micropapillary subtypes: $0.5 ; 95 \%$ Cl: $0.09-2.89 ; p=0.44$; failure time distribution model; HR of solid/micropapillary subtypes: $0.36 ; 95 \% \mathrm{Cl}$ : 0.03-3.8; $p=0.39$; Supplementary Table 6).

\section{Discussion}

To improve prognostic validity for non-small cell lung cancers, the eighth-edition staging system by the AJCC subclassifies the clinical and pathologic T categories in more detail based on the tumor extent; these categories are defined according to measurements of the solid portion size on CT and pathologically invasive component, respectively. ${ }^{24-26}$ In this context, we investigated the prognostic value of the predominant histologic subtype in conjunction with this new staging system in stage I adenocarcinomas. Although the predominant subtype classification is a prognostic factor for lung adenocarcinoma, this subtype was not found to be an independent predictor for OS in multi-aspect survival analyses, including the adjusted log-rank test, multivariable Cox hazard regression analysis, and the mixture cure model. The results were consistent in adenocarcinomas that presented as solid nodules on chest CT.

Murakami et al. ${ }^{17}$ proposed that the histologic subtype was correlated with disease-specific survival, although the classification was not associated with the prognosis in a multivariable Cox regression analysis. Likewise, Ujiie et al. ${ }^{18}$ reported a difference in OS between low (lepidic), intermediate (acinar/papillary), and high (solid/micropapillary) grades. Song et al. ${ }^{8}$ and Hung et al. ${ }^{6}$ suggested that solid/micropapillary and lepidic-predominant subtypes were negative and positive prognostic factors for OS, respectively. However, those studies were performed on the basis of the seventh-edition staging system, and the solid portion size or the invasive component size was not analyzed. $6,8,17,18$ In addition, the nodule type (i.e., the presence of a ground-glass opacity component in tumors), which is one of the pivotal covariates for prognostication in adenocarcinomas, ${ }^{19,20,27}$ was not included in their analyses. Recently, Hattori et al. ${ }^{20}$ applied the eighth-edition staging system in clinical stage I adenocarcinomas and demonstrated differences in OS according to the histologic subtype. Nevertheless, their study had limitations that only a univariable analysis was conducted and the micropapillary-predominant subtype was absent in the study population. ${ }^{20}$ Other publications reporting the prognostic value of histologic subtypes differed from our study in that they included adenocarcinomas of stage II or higher. $4,5,7,9-$ $11,13-16,22,23$

In this study, we performed multi-aspect survival analyses. In principle, Cox proportional hazard regression analysis is based on the assumption that the event will eventually occur and the survival curve will decrease to zero. ${ }^{28}$ It is the conventional method for survival analysis in the lung cancer staging 
system. ${ }^{26}$ In contrast, the mixture cure model assumes that the plateau of the survival curve in long-term follow-up reflects the cure state of patients. ${ }^{29,30}$ Given the presence of long-term censored patients among those with stage I adenocarcinoma, the mixture cure model is applicable, ${ }^{29-31}$ and investigators can separately analyze the predictors for long-term and short-term survival. ${ }^{29,30}$ The consistent statistical results obtained from the multi-aspect survival analyses confirmed the robustness of our observations.

Long-term follow-up (at least 5 years) for the study population is another strength of our study. The median follow-up duration was 81.3 months, which enabled the investigation of a number of events in patients with stage I adenocarcinomas. Due to the homogeneous and sufficient follow-up duration, milestone survival rates at 5 years could be directly described and compared according to the predominant histologic subtypes.

Some limitations of our study should be mentioned. First, we used the clinical T category as an input for the multivariable survival analyses, not the pathologic $T$ category. However, a recent study proposed that the clinical T category may be a better prognostic indicator than the pathological T category. ${ }^{32}$ Second, variants of invasive adenocarcinoma were not included (e.g., invasive mucinous, colloid, fetal, and enteric adenocarcinoma). ${ }^{3}$ In addition, the latest reported histologic subtypes were not assessed. For example, although it is still not included in a formal classification, adenocarcinoma of the cribriform subtype, known as the high-grade acinar subtype, was reported to have poor outcomes similar to those of the solid/micropapillary-predominant subtype. ${ }^{33-36}$ The absence of this concept, as part of the latest classification of histologic subtypes, may have led to an inaccurate histologic classification, as it can be arbitrarily classified as the acinar or solid subtype. ${ }^{5}$ Further studies in a cohort with the latest pathological classification are warranted. Third, the novel IASLC grading system, which is based on a combination of the most predominant pattern and any high-grade histological pattern ( $20 \%$ or more), was not analyzed. ${ }^{5}$ However, Sica's grading system based on two predominant patterns had a comparable discrimination performance to the novel IASLC system according to a validation study. ${ }^{37}$

In conclusion, although the predominant histologic subtype classification is a prognostic factor for lung adenocarcinoma, no association of this classification with OS was observed in stage I adenocarcinoma according to the eighth-edition staging system. Multi-center studies with a large cohort and up-to-date pathologic information are warranted to further validate our results.

\section{Methods}

The Institutional Review Board of Seoul National University Hospital approved this study and waived the requirement for patients' informed consent. All experiments and methods were performed in accordance with relevant guidelines and regulations.

\section{Study Population}


This study was conducted at a single tertiary referral center in South Korea. Between January 2011 and December 2015, patients who underwent surgical resection for pathologic stage I primary lung adenocarcinoma according to the seventh-edition staging system were identified retrospectively through a dedicated search of electronic medical records. ${ }^{38}$ The study population was then determined with the following exclusion criteria: 1) patients who underwent sublobar resection; 2) synchronous or metachronous lung cancer; 3) adenocarcinoma in situ and minimally invasive adenocarcinoma; 4) invasive mucinous adenocarcinoma; 5) pure ground-glass nodule on chest CT; 6) no available survival data; 7) clinical variables not obtainable; 8) adenocarcinoma histologic subtypes not described in pathology reports; and 9) a non-measurable primary tumor on CT. These exclusion criteria were determined to eliminate potential prognostic confounders. Consequently, 587 patients with pathologic stage I lung adenocarcinoma who underwent at least lobectomy were included in this study (Supplementary Fig. 3).

\section{Data Collection}

The following data were obtained from patients' electronic medical records: demographic information (age, sex, history of malignancy other than lung cancer, and smoking status [never smoker, current, or exsmoker]), surgical record (operation date and operation modality), radiologic information on preoperative CT examinations (nodule type [part-solid nodule or solid nodule] and lobar location), and pathologic report (pathologic diagnosis, stage, and predominant histologic subtypes).

The pathologic diagnosis and stage were determined during routine clinical practice, and the specimens were not reviewed specifically for this study. Therefore, the pathological stage was based on the seventh edition of the AJCC staging system for lung cancer, ${ }^{38}$ as the participants underwent surgery before the release of the eighth edition of the staging system. ${ }^{25,26}$ Thus, we measured the solid component size of each tumor on CT scans to determine the clinical T category according to the eighth edition of the staging system, ${ }^{25,26}$ which was used as a covariate in the multivariable survival analyses. Indeed, a recent study reported that the solid portion size on CT demonstrated a better prognostic correlation than the invasive component size. ${ }^{32}$

The longest diameter of the solid portion was measured on the lung window setting (window width, 1500 $\mathrm{HU}$; level, -700 HU). All measurements were performed in the axial plane using an electronic caliper by one of the two trained radiology technicians (either M.L. with 10 years of research experience in chest CT or J.Y.J. with 3 years of research experience in chest CT) under the supervision of a board-certified thoracic radiologist (H.K. with 10 years of CT experience). Reviewers were blinded to the pathologic diagnosis, stage, and histologic subtypes, but the lobar location was provided.

In this study, the primary endpoint was overall survival (OS), which was measured from the date of surgery for the corresponding lung cancer to the date of death from any cause (i.e., all-cause mortality). The censoring time was at March 18,2021, and the survival status and date of death were obtained from the database of the Ministry of the Interior and Safety. ${ }^{39}$ 


\section{Pathologic Diagnosis}

In this study, the 2011 IASLC/ATS/ERS classification of lung adenocarcinoma was applied. ${ }^{3}$ That is, nonmucinous invasive adenocarcinomas were categorized by their predominant architecture patterns (lepidic, acinar, papillary, micropapillary, or solid-predominant subtype). ${ }^{3,4}$ The final pathologic diagnosis was adjudicated by the board-certified attending pathologists of our hospital as part of their routine clinical practice. Then, according to Sica's three-tier grading system, ${ }^{40}$ adenocarcinomas were categorized into three groups: 1) lepidic-predominant, 2) acinar/papillary-predominant, and 3) solid/micropapillarypredominant subtypes. ${ }^{3-5,41}$

In $26 \%$ (153 of 587 ) of the study population, mixed subtype adenocarcinoma was reported according to the 2004 World Health Organization classification, ${ }^{42}$ and the predominant subtype was not described in the pathology reports. In these patients, the three-tier grading was determined following the histologic hierarchy. ${ }^{5}$ Specifically, if the solid or micropapillary subtype was noted in the mixed subtype, it was classified as the solid/micropapillary subtype.

\section{CT Examinations}

CT scans were performed using eight different scanners produced by four manufacturers (Brilliance 64, Ingenuity, and iCT, Philips Healthcare, Best, Netherlands; LightSpeed Ultra and Discovery CT750HD, GE Healthcare, Waukesha, WI, USA; Somatom Sensation 16 and Definition, Siemens Healthcare, Forchheim, Germany; Aquilion One, Toshiba Medical Systems [now Canon Medical Systems], Otawara, Japan). Since this study was performed at a tertiary medical center, which operates multiple CT scanners that are purchased from various vendors, heterogeneity in imaging acquisition was inevitable due to the retrospective nature of this study. All patients underwent CT scans from the lung apex to base at suspended maximum inspiration. The scans were performed at $120 \mathrm{kVp}$ and approximately 20 to 200 $\mathrm{mAs}$ with or without the automatic exposure control of each vendor. The CT scans were reconstructed with a slice thickness $\leq 5 \mathrm{~mm}$. For patients with part-solid nodules, the slice thickness was less than or equal to $1.5 \mathrm{~mm}$.

\section{Statistical Analysis}

Across the three histologic groups, the Kruskal-Wallis test for continuous variables and the Pearson chisquare test or Fisher exact test for categorical variables were performed for the patient and tumor characteristics.

To investigate the prognostic value of the predominant histologic subtype for OS, we performed threelevel survival analyses: 1) pairwise comparisons among the three histological groups (lepidicpredominant, acinar/papillary-predominant, and solid/micropapillary-predominant subtypes) using unadjusted and adjusted log-rank tests; for the adjusted log-rank test, variables of age, sex, cancer history, smoking status, nodule type, and clinical T category (cT1 mi/cT1a, cT1b, cT1, and cT2) were taken into account using inverse probability weighting; ${ }^{43}$ 2) multivariable Cox proportional hazard regression 
analysis with the histologic subtype and other input variables used for the adjusted log-rank test; and 3) a multivariable mixture cure model including a cure probability model for patients with long-term survival and a failure time distribution model for those who died. ${ }^{29,30}$

For a study population in which a subset of patients are long-term survivors, the mixture cure model can be used to investigate the heterogeneity between patients with two distinct prognoses and to assess their predictors, respectively (a cure probability model for long-term survival and a failure time distribution model for short-term survival). ${ }^{29,30}$ The relevance of this analysis depends on whether the survival curve has a plateau after long-term follow-up. ${ }^{29,30}$ Since early-stage, resected lung adenocarcinomas show a very long-term censored survival time, the cure model was deemed applicable to our study. ${ }^{31}$

For the mixture cure model, the same covariates were used as in the multivariable Cox regression, but the clinical T categories were collapsed into three groups (i.e., cT1 mi/cT1a/cT1b, cT1c, and cT2) and the histologic subtype category was also collapsed into two groups (lepidic/acinar/papillary vs. solid/micropapillary) to estimate confidence intervals (Cls) for the hazard ratio (HR) and odds ratio (OR) robustly.

We then performed subgroup analyses to evaluate the prognostic association of the histologic subtype with OS solely in adenocarcinomas manifesting as solid tumors on CT. Because of the small number of adenocarcinomas with cT1mi/cT1a and the lepidic-predominant subtype, they were combined with cT1b and the acinar/papillary-predominant subtypes, respectively. In addition, sensitivity analyses were performed after excluding those with mixed subtype adenocarcinoma.

All statistical analyses were conducted using R software, version 4.0.4 (R Project for Statistical Computing, Vienna, Austria; packages: RISCA, smcure, survival, and survminer), and $p<0.05$ indicated statistical significance.

\section{Abbreviations}

OS

overall survival

IASLC/ATS/ERS

International Association for the Study of Lung Cancer/American Thoracic Society/European Respiratory Society

AJCC

American Joint Commission on Cancer

\section{Declarations}

\section{Acknowledgment}


The authors would like to acknowledge Andrew Dombrowski, Ph.D. (Compecs, Inc.) for his assistance in improving the use of English in this manuscript.

\section{Author contributions}

J.H.L.: Investigation, Data Curation, Writing - Original Draft, Writing - Review \& Editing.

H.K.: Conceptualization, Methodology, Formal analysis, Resources, Data Curation, Writing - Original Draft, Writing - Review \& Editing, Visualization, Supervision, Funding acquisition.

C.M.P, Y.K.J, D.H.C., Y.T.K., J.M.G.: Investigation, Resources, Writing - Original Draft, Writing - Review \& Editing

\section{Data availability statement}

The datasets generated during and/or analysed during the current study are not publicly available because the datasets composed of patients from Seoul National University Hospital, which is attributed to the hospital, but are available from the corresponding author on reasonable request.

\section{Conflicts of interest}

Activities related to the present article: none.

Activities not related to the present article: H.K. received a research grant from Lunit; holds stock in Medical IP. C.M.P. received research grants from Lunit and Coreline Soft; holds stock in Promedius and stock options in Lunit and Coreline Soft. J.M.G. received research grants from Lunit, INFINITT Healthcare, Dongkook Lifescience, and LG electronics. Y.T.K is a consultant of Ignite Thoracic Council (Johnson and Johnson).

\section{Source of funding}

This study was supported by the Basic Science Research Program through the National Research Foundation of Korea (NRF), funded by the Ministry of Science and ICT (grant number: NRF2020R1C1C1003684), however, the funder had no role in the study design; in the collection, analysis, and interpretation of the data; in the writing of the report; and in the decision to submit the article for publication.

\section{References}

1. Sung, H. et al. Global Cancer Statistics 2020: GLOBOCAN Estimates of Incidence and Mortality Worldwide for 36 Cancers in 185 Countries. CA Cancer J Clin. 71, 209-249 (2021).

2. Siegel, R.L., Miller, K.D., Jemal, A. Cancer statistics, 2020. CA Cancer J Clin. 70, 7-30 (2020). 
3. Travis, W.D. et al. International association for the study of lung cancer/american thoracic society/european respiratory society international multidisciplinary classification of lung adenocarcinoma. J Thorac Oncol. 6, 244-285 (2011).

4. Warth, A. et al. The novel histologic International Association for the Study of Lung Cancer/American Thoracic Society/European Respiratory Society classification system of lung adenocarcinoma is a stage-independent predictor of survival. J Clin Oncol. 30, 1438-1446 (2012).

5. Moreira, A.L. et al. A grading system for invasive pulmonary adenocarcinoma: a proposal from the International Association for the Study of Lung Cancer Pathology Committee. J Thorac Oncol. 15, 1599-1610 (2020).

6. Hung, J.-J. et al. Prognostic value of the new International Association for the Study of Lung Cancer/American Thoracic Society/European Respiratory Society lung adenocarcinoma classification on death and recurrence in completely resected stage I lung adenocarcinoma. Ann Surg. 258, 1079-1086 (2013).

7. Russell, P. A. et al. Correlation of mutation status and survival with predominant histologic subtype according to the new IASLC/ATS/ERS lung adenocarcinoma classification in stage III (N2) patients. J Thorac Oncol. 8, 461-468 (2013).

8. Song, Z. et al. Prognostic value of the IASLC/ATS/ERS classification in stage I lung adenocarcinoma patients-based on a hospital study in China. Eur J Surg Oncol. 39, 1262-1268 (2013).

9. Tsuta, K. et al. The utility of the proposed IASLC/ATS/ERS lung adenocarcinoma subtypes for disease prognosis and correlation of driver gene alterations. Lung cancer. 81, 371-376 (2013).

10. Yoshizawa, A. et al. Validation of the IASLC/ATS/ERS lung adenocarcinoma classification for prognosis and association with EGFR and KRAS gene mutations: analysis of 440 Japanese patients. J Thorac Oncol. 8, 52-61 (2013).

11. Campos-Parra, A. D. et al. Relevance of the novel IASLC/ATS/ERS classification of lung adenocarcinoma in advanced disease. Eur Respir J. 43, 1439-1447 (2014).

12. Cha, M. J. et al. Micropapillary and solid subtypes of invasive lung adenocarcinoma: clinical predictors of histopathology and outcome. J Thorac Cardiovasc Surg. 147, 921-928. e922 (2014).

13. Hung, J.-J. et al. Predictive value of the International Association for the Study of Lung Cancer/American Thoracic Society/European Respiratory Society classification of lung adenocarcinoma in tumor recurrence and patient survival. J Clin Oncol. 32, 2357-2364 (2014).

14. Mansuet-Lupo, A. et al. The new histologic classification of lung primary adenocarcinoma subtypes is a reliable prognostic marker and identifies tumors with different mutation status: the experience of a French cohort. Chest. 146, 633-643 (2014).

15. Yanagawa, N. et al. The correlation of the International Association for the Study of Lung Cancer (IASLC)/American Thoracic Society (ATS)/European Respiratory Society (ERS) classification with prognosis and EGFR mutation in lung adenocarcinoma. Ann Thorac Surg. 98, 453-458 (2014).

16. Lee, G. et al. Clinical impact of minimal micropapillary pattern in invasive lung adenocarcinoma. Am J Surg Pathol. 39, 660-666 (2015). 
17. Murakami, S. et al. Prognostic value of the new IASLC/ATS/ERS classification of clinical stage IA lung adenocarcinoma. Lung cancer. 90, 199-204 (2015).

18. Ujiie, H. et al. Solid predominant histologic subtype in resected stage I lung adenocarcinoma is an independent predictor of early, extrathoracic, multisite recurrence and of poor postrecurrence survival. $\mathrm{J}$ Clin Oncol. 33, 2877 (2015).

19. Aokage, K. et al. Influence of ground glass opacity and the corresponding pathological findings on survival in patients with clinical stage I non-small cell lung cancer. J Thorac Oncol. 13, 533-542 (2018).

20. Hattori, A. et al. Distinct clinicopathologic characteristics and prognosis based on the presence of ground glass opacity component in clinical stage IA lung adenocarcinoma. J Thorac Oncol. 14, 265275 (2019).

21. Matsubara, T. et al. Clinical and Prognostic Significance of the Epithelial-Mesenchymal Transition in Stage IA Lung Adenocarcinoma: A Propensity Score-Matched Analysis. Clin Lung Cancer. 20, e504e513 (2019).

22. Qiu, J.H. et al. Optimised architecture-based grading system as an independent prognostic factor in resected lung adenocarcinoma. J Clin Pathol. 0:1-9. https://doi.org/10.1136/jclinpath-2020-207104 [Online ahead of print] (2020).

23. Westaway, D. D. et al. The International Association for the Study of Lung Cancer/American Thoracic Society/European Respiratory Society grading system has limited prognostic significance in advanced resected pulmonary adenocarcinoma. Pathology. 45, 553-558 (2013).

24. Amin, M.B. et al. AJCC Cancer Staging Manual. 8th ed. Chicago, IL. (Springer International Publishing, 2017).

25. Goldstraw, P. et al. The IASLC lung cancer staging project: proposals for revision of the TNM stage groupings in the forthcoming (eighth) edition of the TNM classification for lung cancer. J Thorac Oncol. 11, 39-51 (2016).

26. Travis, W. D. et al. The IASLC lung cancer staging project: proposals for coding T categories for subsolid nodules and assessment of tumor size in part-solid tumors in the forthcoming eighth edition of the TNM classification of lung cancer. J Thorac Oncol. 11, 1204-1223 (2016).

27. Ye, T. et al. Lung adenocarcinomas manifesting as radiological part-solid nodules define a special clinical subtype. J Thorac Oncol. 14, 617-627 (2019).

28. Wu, Y., Lin, Y., Lu, S.-E., Li, C.-S. \& Shih, W. J. Extension of a Cox proportional hazards cure model when cure information is partially known. Biostatistics. 15, 540-554 (2014).

29. Othus, M., Barlogie, B., LeBlanc, M. L. \& Crowley, J. J. Cure models as a useful statistical tool for analyzing survival. Clin Cancer Res. 18, 3731-3736 (2012).

30. Amico, M. \& Van Keilegom, I. Cure models in survival analysis. Annu Rev Stat Appl. 5, 311-342 (2018).

31. Kim, H., Goo, J. M., Kim, Y. T. \& Park, C. M. Validation of the eighth edition clinical T categorization system for clinical stage IA, resected lung adenocarcinomas: prognostic implications of the ground- 
glass opacity component. J Thorac Oncol. 15, 580-588 (2020).

32. Funai, K., Kawase, A., Mizuno, K., Koyama, S. \& Shiiya, N. tumor, node, and metastasis T-stage prognosis discrepancies: Solid component diameter predicts prognosis better than invasive component diameter. Cancers. 12, 1577 (2020).

33. Warth, A. et al. Prognostic impact and clinicopathological correlations of the cribriform pattern in pulmonary adenocarcinoma. J Thorac Oncol. 10, 638-644 (2015).

34. Moreira, A. L., Joubert, P., Downey, R. J. \& Rekhtman, N. Cribriform and fused glands are patterns of high-grade pulmonary adenocarcinoma. Hum Pathol. 45, 213-220 (2014).

35. Kadota, K. et al. The cribriform pattern identifies a subset of acinar predominant tumors with poor prognosis in patients with stage I lung adenocarcinoma: a conceptual proposal to classify cribriform predominant tumors as a distinct histologic subtype. Mod Pathol. 27, 690-700 (2014).

36. Kadota, K. et al. Cribriform subtype is an independent predictor of recurrence and survival after adjustment for the eighth edition of TNM staging system in patients with resected lung adenocarcinoma. J Thorac Oncol. 14, 245-254 (2019).

37. Rokutan-Kurata, M. et al. Brief Report: Validation Study of the IASLC Histological Grading System of Invasive Lung Adenocarcinoma1. J Thorac Oncol. https://doi.org/10.1016/j.jtho.2021.04.008 [Online ahead of print] (2021).

38. Goldstraw, P. et al. The IASLC Lung Cancer Staging Project: proposals for the revision of the TNM stage groupings in the forthcoming (seventh) edition of the TNM Classification of malignant tumours. J Thorac Oncol. 2, 706-714 (2007).

39. Jung, K.-W., Won, Y.-J., Kong, H.-J. \& Lee, E. S. Cancer statistics in Korea: incidence, mortality, survival, and prevalence in 2015. Cancer Res Treat. 50, 303-316 (2018).

40. Sica, G. et al. A grading system of lung adenocarcinomas based on histologic pattern is predictive of disease recurrence in stage I tumors. Am J Surg Pathol. 34, 1155-1162 (2010).

41. Travis, W. D. et al. The 2015 World Health Organization classification of lung tumors: impact of genetic, clinical and radiologic advances since the 2004 classification. J Thorac Oncol. 10, 12431260 (2015).

42. Travis WD, B. E., Müller-Hermelink HK, Harris CC. Pathologyand Genetics: Tumours of the Lung, Pleura, Thymus and Heart. (Lyon: IARC, 2004).

43. Xie, J. \& Liu, C. Adjusted Kaplan-Meier estimator and log-rank test with inverse probability of treatment weighting for survival data. Stat Med. 24, 3089-3110 (2005).

\section{Figures}




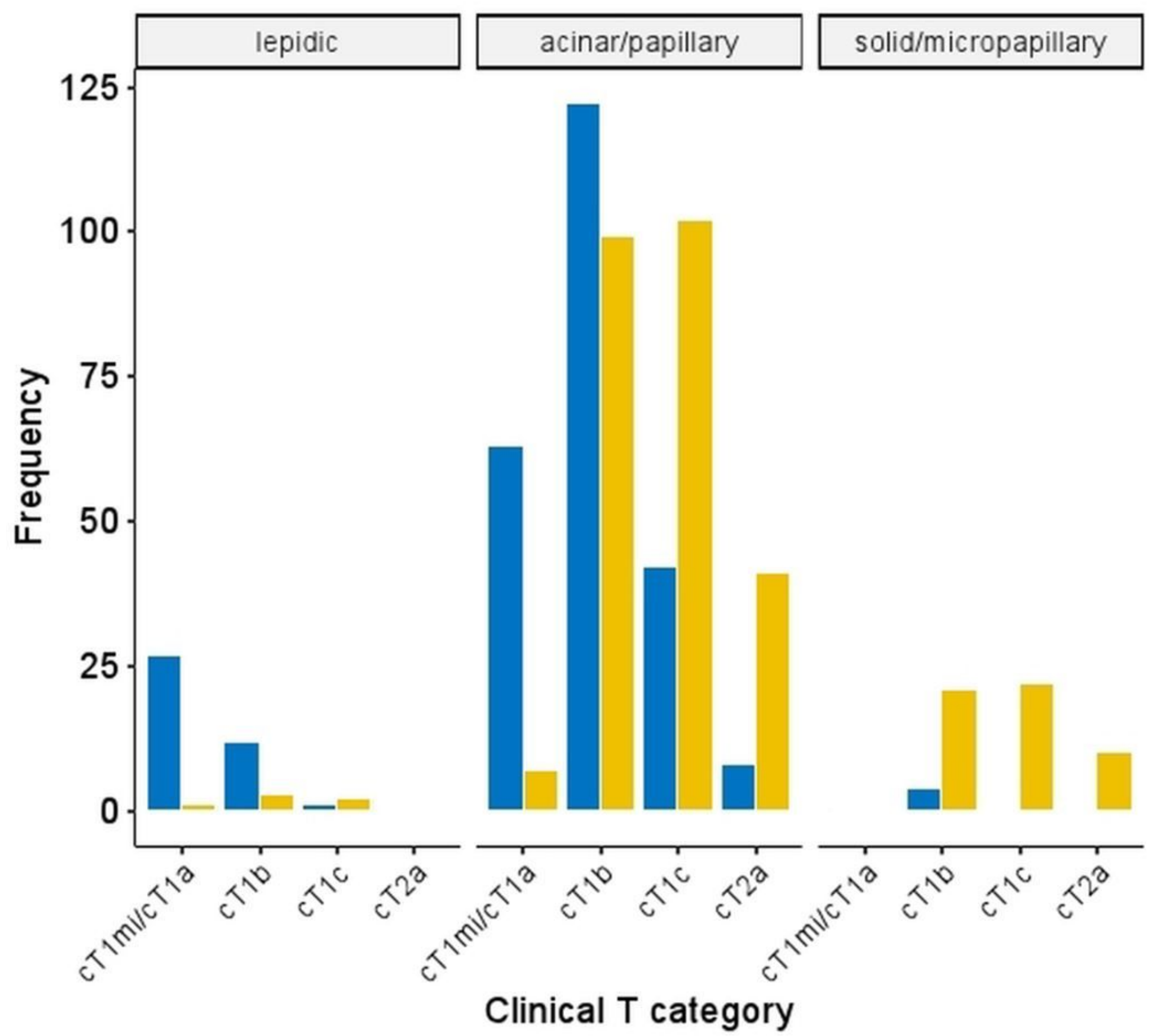

Figure 1

Bar plot for the number of adenocarcinomas according to the predominant histologic subtype, clinical T category, and nodule type. 

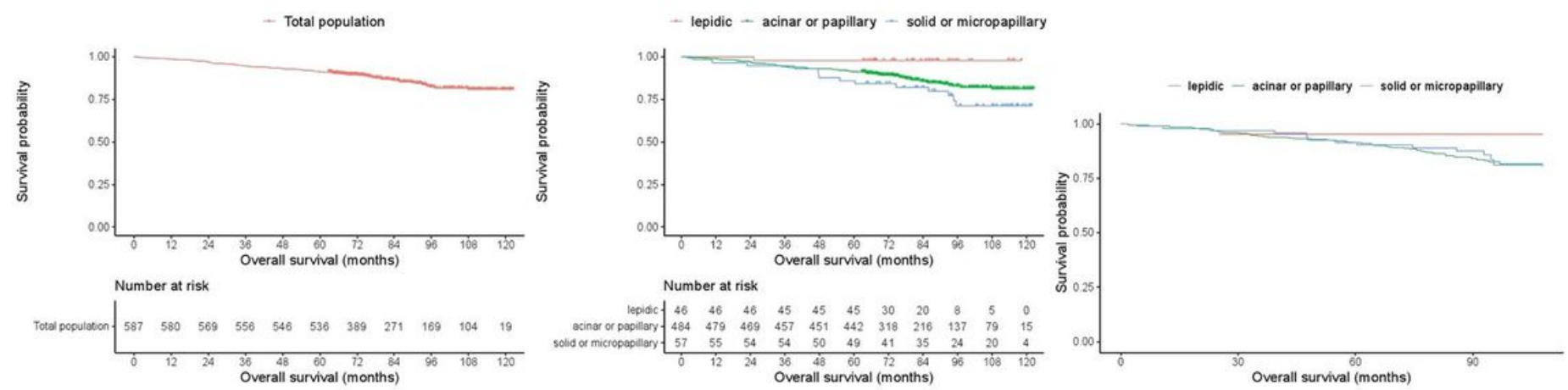

Figure 2

Survival curves for overall survival of patients with stage I adenocarcinoma.

(A) Total study population. Note the censored patients at long-term follow-up and a plateau at the end.

(B) Unadjusted survival curves according to the predominant histologic subtype.

(C) Adjusted survival curves stratified by the predominant histologic subtype (adjusted for age, sex, cancer history, smoking status, nodule type, and clinical T category).

\section{Supplementary Files}

This is a list of supplementary files associated with this preprint. Click to download.

- SupplementaryMaterials.pdf 